\title{
TUMOR DE CÉLULAS DE LA GRANULOSA: DIAGNÓSTICO, TRATAMIENTO HORMONAL E INTERVENCIÓN QUIRÚRGICA EN YEGUA CRIOLLA COLOMBIANA.
}

\section{GRANULOSA CELL TUMOR: DIAGNOSTIC, HORMONAL TREATMENT AND SURGICAL INTERVENTION IN MARE CREOLE.}

RUIZ, V. ANDRÉS ${ }^{1}$ MV., RIVERA, C. LUIS GABRIEL ${ }^{*}$ MVZ., CALDERON, H. CARLOS EDUARDO ${ }^{3}$., FRANCO, H. ALEXER ${ }^{3}$., GOMEZ, DAMIAN ${ }^{3}$.

${ }^{1}$ Profesor de la Universidad de la Salle, Bogotá D.C, Colombia.

2 Estudiante de Maestría en la Universidad Estadual Paulista "Júlio de mesquita filho" (UNESP), Facultad de ciencias agrarias y veterinarias, Departamento de Patología Veterinaria, Programa de Posgraduación de Medicina Veterinaria, Jaboticabal, SP, Brasil.

${ }^{3}$ Estudiante de la Universidad de la Amazonia, Facultad de Ciencias Agrarias y Veterinarias, Programa de Medicina Veterinaria y Zootecnia, Florencia, Caquetá, Colombia

Correspondencia:* Igriveramvz@gmail.com

Recibido: 25-10-2013; Aceptado: 14-11-2013.

\section{Resumen}

Graves problemas reproductivos se han asociado a tumores ováricos en yeguas. Dentro de estos el Tumor de Células de la Granulosa (TCG) sigue siendo el más diagnosticado, aunque sólo representa el $2.5 \%$ de todos los tumores del equino. El siguiente es un estudio de caso de TCG en una yegua criolla adulta que manifestó comportamiento similar al macho, virilismo y anestro. Se utilizó el examen ultrasonográfico para identificar el tumor y se administró $\mathrm{GnRH}$ como tratamiento hormonal; en semanas posteriores se procedió a la ovariectomia como tratamiento quirúrgico. El examen histopatológico del tejido neoplásico evidenció proliferación celular con características morfológicamente semejantes a las células de la granulosa, siendo el diagnóstico definitivo TCG. Se concluye que el tratamiento hormonal y quirúrgico fue satisfactorio, lo cual se pudo comprobar por el cese de comportamientos anormales y el retorno de la ciclicidad del ovario restante, se espera que en meses posteriores la yegua pueda ser utilizada nuevamente para reproducción.

Palabras Claves: Ovariectomia, Tumor de Células de la granulosa, Reproducción, Yegua.

\section{Abstract}

Severe reproductive problems in mares are related to ovarian tumors. Among these, the Granulosa Cell Tumor (GCT) is the most commonly diagnosed, although it represents only $2.5 \%$ of all the tumors in horses. The following 
investigation is a case study of GCT in one creole adult mare, which presented a similar behavior as the male, virilism and anestrus. An ultrasonographic examination was used to identify the tumor and $\mathrm{GnRH}$ was administered as hormonal treatment; in the following weeks an ovariectomy was done as a surgical treatment. The histopathologic examination of the neoplasic tissue evidenced cellular proliferation with similar morphologic characteristics as the granulosa cells, which is the definitive diagnosis for GCT. The hormonal and surgical treatment was satisfactory, verified by the cessation of abnormal behaviors and by the return of the cyclicity of the remaining ovary. It is expected that in the following months the mare can be used again to reproduction.

Keywords: Ovariectomy, Granulosa Cell Tumor, Reproduction, Mare.

\section{Introducción}

El ovario es un órgano extremadamente complejo desde el punto de vista histiogenético (LIMA et al., 2011); anatómicamente muestra en su región interna una zona medullaris (laxa, rica en vasos sanguíneos) y en su región externa una zona parenchymatosa, densamente estructurada con un gran número de formaciones funcionales (KÖNING et al., 2005). La diversidad de tejidos en su interior justifican el origen de diferentes tipos de tumores (LIMA et al., 2011).

Procesos neoplásicos ocurren con relativa frecuencia en el ovario; son observados en todas las especies de animales domésticos, siendo más comunes en hembras bovinas, caninas y equinas (LIMA et al., 2011). Las neoplasias ováricas son clasificadas con base en su morfología, hallazgos clínicos, capacidad o potencialidad de malignidad y su histogénesis (FAGUNDES y LIMA, 2003).

Dentro de las neoplasias primarias del órgano se encuentran tres categorías principales: neoplasias de las células germinativas ( $p$. ej. Teratoma, disgerminoma), del estroma gonadal (p. ej. Tumor de células de la granulosa, tecoma) y del epitelio (p. ej. Adenoma papilífero, carcinoma papilífero) (MACLACHLAN y KENNEDY, 2004; FOSTER, 2009).

Por otro lado, así como las neoplasias, existen alteraciones en el ovario que pueden generar anestro prolongado y reducción en la tasa de fertilidad (MCCUE, 2000). En yeguas por ejemplo, el diagnóstico de anormalidades reproductivas se observa principalmente por procesos inflamatorios del útero (como en la endometritis bacteriana) y en las fallas ováricas (por folículos hemorrágicos o anovulatorios) (MCCUE, 2009; OTALVARO et al., 2009).

Es indispensable para el médico veterinario realizar un examen clínico detallado que permita determinar la causa de estos problemas reproductivos, recordando que en su etiología también pueden estar involucrados procesos proliferativos, los cuales pocas veces son reportados, debido a la inadecuada inspección del tracto reproductivo, aun después de efectuar la necropsia del animal (FAGUNDES y LIMA, 2003). 
Entre los procesos proliferativos más comunes del ovario en yeguas se encuentra el Tumor de Células de la Granulosa (TCG), que corresponde aproximadamente a un $85 \%$ de las neoplasias del tracto reproductivo y el $2.5 \%$ de todos los tumores en esta especie (MCCUE et al., 2006); su importancia clínica radica en la producción de hormonas esteroides que pueden interfieren con la función reproductiva de la hembra (LIMA et al., 2011).

Las células de la granulosa se ubican en una camada que circundan internamente el folículo, están involucradas en la producción de inhibina y junto con las células de la teca en la producción de estradiol (FRAZER, 2003). En algunas ocasiones el TCG está compuesto por un número significativo de células tecales, razón por la cual, es denominado Tumor de Células de la Granulosa y de la Teca por varios autores (MAURICE, 2005; SCHLAFER y MILLER, 2007; MCKINNON y BARKER 2010). Con frecuencia el TCG contiene células tecales que pueden aumentar la producción de testosterona, asociada con la ninfomanía, virilismo o anestro en las yeguas afectadas (Schlafer y Miller 2007, Lima et al., 2011). El diagnóstico que realiza la clasificación del tumor depende de los niveles sanguíneos hormonales principalmente de: testosterona, inhibina y progesterona; así como del examen ultrasonográfico e histopatológico (LEBLANC et al., 2003).

A continuación se relata el caso clínico de una yegua adulta que presenta TCG. En el estudio se describe el historial reproductivo del animal, las herramientas diagnósticas utilizadas para demostrar la presencia del tumor, además del tratamiento hormonal y quirúrgico efectuado para su remoción. Todo lo anterior tiene como objetivo brindar un protocolo de diagnóstico y tratamiento a la hora de abordar esta neoplasia, el cual va dirigido a médicos veterinarios involucrados en el área de reproducción.

\section{Descripción del caso}

El 02 de enero de 2012, se realiza la consulta de una yegua criolla de trocha y galope colombiano en el criadero de San marcos en el municipio de Tenjo (Cundinamarca, Colombia). La yegua de color zaino, 9 años de edad, con un peso de $320 \mathrm{~kg}$ (condición corporal de 3.5 en la escala de 1 a 5) se observa con buen estado de salud.

Dentro de los antecedentes, la hembra realizó su último parto cinco meses antes de la consulta y produjo una cría por transferencia de embriones. Su ciclo reproductivo siempre se mantuvo estable, con un promedio de intervalo entre ovulaciones de 20 días. La última vez que se sirvió fue el 15 de noviembre de 2011, tres semanas después el personal de la finca manifestó que la yegua había entrado de nuevo en celo, por lo cual se realizó la consulta a principios de enero.

Durante la consulta, a la palpación rectal se observó: ovario derecho de aproximadamente $6 \mathrm{~cm}$ de diámetro con una estructura semejante a un folículo anovulatorio; el ovario izquierdo se encontró estático de unos $3 \mathrm{~cm}$ de diámetro. Días después se hizo un seguimiento por examen ultrasonográfico al posible proceso proliferativo del ovario derecho. 


\section{Examen Ultrasonográfico}

El 16 de enero de 2012, se evidenció por examen ultrasonográfico transrectal con sonda linear 5.0 y $7.5 \mathrm{MHz}$ (Piemedical- $200^{\circledR}$ ) un posible tumor de células de la granulosa de $8 \mathrm{~cm}$ de diámetro en ovario derecho. El ovario izquierdo se observó atrofiado (de 2,8 cm de diámetro), con superficie lisa y completamente estático. Para el 13 de febrero de 2012, la masa tumoral en ovario derecho alcanzó los $20 \mathrm{~cm}$ de diámetro. En la Figura 1 se observa el ovario derecho aumentado de tamaño, poliquístico y con forma de panal de abeja.

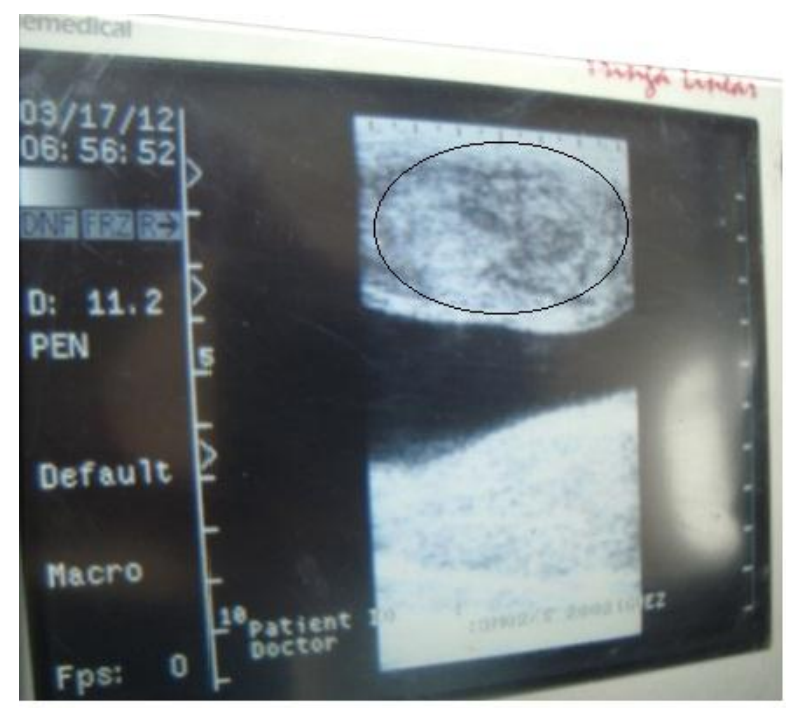

Figura 1. Ultrasonografía de ovario derecho de yegua criolla. Obsérvese la masa tumoral con forma de panal de abeja por los múltiples quistes (encerrada en círculo). El tamaño de la neoplasia es de $20 \mathrm{~cm}$ de diámetro, no abarcable en toda su extensión por el transductor; la parte central anecogénica es la vejiga dilatada.

Durante los días que se realizaron los exámenes ecográficos, la hembra comenzó a perder peso progresivamente. Se observó inquieta en la pesebrera y cuando se enviaba a potrero junto con otros caballos manifestaba virilismo, montando las yeguas que se encontraban en celo; igualmente presentó dominancia y agresividad hacia los demás animales e incluso hacia el personal del criadero.

\section{Tratamiento hormonal}


El 5 de mayo de 2012 se aplicó una dosis única de $\mathrm{GnRH}$ a razón de $2.2 \mathrm{mg}$ IM, esto con el fin de permitir la regresión del tumor y continuar en semanas posteriores con el tratamiento quirúrgico.

\section{Procedimiento Quirúrgico: Ovariectomia.}

Antes de la intervención quirúrgica se administró flunixin meglumina $(1.1 \mathrm{mg} / \mathrm{kg}$ PV IV), xilacina $(0.8 \mathrm{mg} / \mathrm{kg}$ PV IV) y butorfanol $(0.01 \mathrm{mg} / \mathrm{kg}$ PV IV) como medicación preanestésica; fue realizado un bloqueo regional infiltrativo con lidocaína 2\% (100 ml) alcanzando tejido subcutáneo y muscular en la zona del abordaje quirúrgico. La incisión abdominal fue efectuada de forma paramediana diagonal, al lado respectivo donde se identificó el ovario alterado.

Macroscópicamente se observó el ovario derecho con forma circular, de superficie irregular, con $14 \mathrm{~cm}$ de diámetro. Presentaba color amarillo con depósito de fibrina y consistencia sólida (Figura 2 A). Al corte se observó múltiples lesiones quísticas con líquido seroso sanguinolento (Figura 2 B).
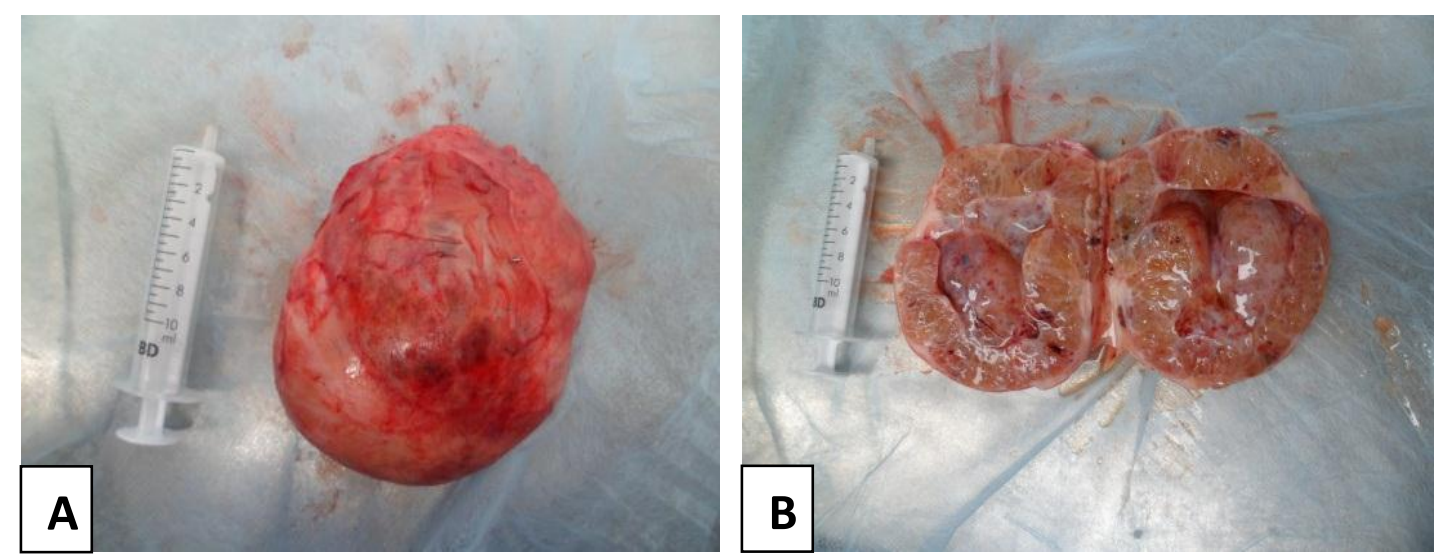

Figura 2 A. Ovario derecho aumentado de tamaño por el TCG. B. Corte Sagital del ovario derecho. Obsérvese las lesiones quísticas producidas por el tumor.

En el postoperatorio se prescribió flunixin meglumina en dosis de $1,1 \mathrm{mg} / \mathrm{kg} \mathrm{PV}$, IM por 4 días; además de la aplicación intravenosa de Penicilina sódica a una dosis de $40000 \mathrm{Ul} / \mathrm{kg}$ PV y Gentamicina a $6.6 \mathrm{mg} / \mathrm{kg}$ PV, los dos antibióticos se administraron cada 12 horas por 4 días.

\section{Examen Histopatológico}

Se colectaron muestras de diferentes áreas del tumor para el examen histopatológico de acuerdo con FRAZER (2003); estas se enviaron al laboratorio en formalina buferada al 10\%. Después de ejecutarse el proceso de deshidratación, diafanización y parafinización, se realizaron cortes histológicos 
en el micrótomo automático (Leica $2155^{\circledR}$ ) de $3 \mu \mathrm{m}$ de espesor; posteriormente estos cortes fueron teñidos por la técnica de Hematoxilina y Eosina.

En el Microscopio óptico se observaron células redondas con citoplasma escaso y esinofílico; núcleos redondos en el centro de la célula, con cromatina finamente granular (Figura 4). Estas presentaban el mismo padrón que una célula de la granulosa en un folículo terciario.

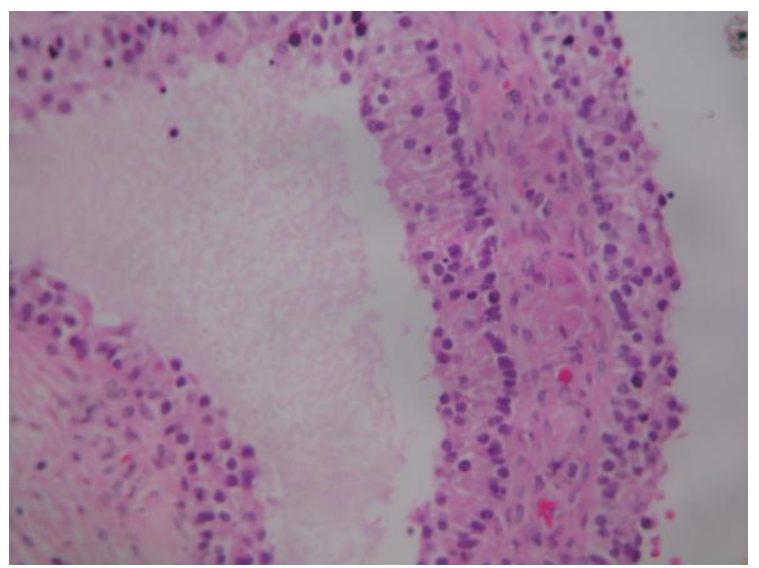

Figura 4. Tumor de células de la granulosa. Área quística donde se observan células de la granulosa con núcleos hipercromáticos. H-E 40 X.

El diagnóstico definitivo fue un Tumor de células de la granulosa en ovario derecho. El 01 de Septiembre del 2011, se realizó un nuevo examen ultrasonográfico y se observó el ovario restante de $3 \mathrm{~cm}$ de diámetro, completamente estático.

\section{Discusión}

A pesar de la baja ocurrencia de neoplasias ováricas en animales domésticos, estas poseen un gran interés desde el punto de vista reproductivo, porque pueden generar modificaciones hormonales y producir perdida de la ciclicidad e incompatibilidad con la gestación. En yeguas varios problemas reproductivos se han asociado a tumores del ovario. El TCG sigue el más diagnosticado, aunque sólo representa el $2.5 \%$ de todos los tumores del equino (MCCUE et al., 2006; MAURICE, 2006). Surge del estroma de los cordones sexuales y posee alto potencial de secretar hormonas esteroides al igual de otros tumores del mismo origen (MACLACHLAN y KENNEDY, 2004; SCHLAFER y MILLER 2007).

En el TCG existen factores predisponentes como la edad y el estado reproductivo; Según MCKINNON y BARKER (2010) típicamente se presenta en yeguas de 5 a 9 años de edad, algunos meses después de haber parido (características que también fueron observadas en la yegua en estudio). Sin embargo, en algunos casos se ha observado en potrancas y en yeguas en gestación (FAGUNDES y LIMA, 2003; PINTO, 2004). 
En el presente estudio, el examen ultrasonográfico evidenció una masa tumoral lobulada semejante a un panel de abejas por los múltiples quistes, concordado con lo observado por FRAZER (2003) y MORA (2004); mientras que el ovario izquierdo se encontró inactivo y atrofiado. Según MCKINNON y BARKER (2010) la identificación de un ovario aumentado de tamaño, poliquístico o una masa solida acompañada de inactividad ovárica contralateral es casi patognomónica de TCG. No obstante, SCHLAFER y MILLER (2007) aseguran que la ultrasonografía no debe ser usada como herramienta para el diagnóstico definitivo del tumor, porque este debe ser diferenciado con otras neoplasias ováricas.

En el TCG generalmente las yeguas afectadas presentan algunos problemas de comportamiento asociados a ninfomanía, virilismo o anestro (FRAZER, 2003; LIMA et al., 2011). La yegua del presente estudio manifestó un comportamiento similar a un semental, montando otras yeguas en celo y siendo muy agresiva con otros animales; este comportamiento también ha sido reportado por (INFANTE, 1998; MAURICE, 2006; HARTWIG, 2009). Según OTALVARO et al., (2009), el comportamiento similar al macho también ha sido identificado en otros problemas del ovario como en los folículos anovulatorios hemorrágicos o luteinización de folículos al comienzo de la preñez.

MCCUE (2000), LEBLANC et al., (2003) y MAURICE (2006) sugieren realizar siempre que sea posible una prueba de los niveles sanguíneos de testosterona e inhibina, ya que los signos clínicos observados en yeguas con TCG dependen principalmente de estas dos hormonas. En el estudio no se detectaron los niveles hormonales en que se encontraba la yegua, pero es muy probable que los niveles de testosterona estuvieran por encima de $100 \mathrm{pg} / \mathrm{mL}$ en plasma, por el comportamiento similar al macho; tal y como lo afirman MORA et al., (2004) y SCHLAFER y MILLER (2007).

Según INFANTE (1998), FRAZER (2003) y MAURICE (2006), los altos niveles de testosterona e inhibina parecen ejercer un efecto de retroalimentación negativa sobre la secreción de las gonadotropinas desde la hipófisis, ocurriendo inactividad y la atrofia del ovario contralateral. Sin embargo, SCHLAFER y MILLER (2007) argumenta que la relación entre la testosterona y la atrofia del ovario contralateral no es convincente porque en otros tumores que se produce un aumento de esta hormona no se ha observado esta adaptación celular.

En un estudio realizado por MCCUE (1992) con 39 yeguas afectadas por TCG, se determinó un aumento en la producción de inhibina en el $87.2 \%$ (34/39) de los individuos; por su parte los niveles de testosterona aumentaron en el $53.8 \%$ de la población. Esta la razón por la cual muchos autores no optan por usar la testosterona para el diagnóstico de TCG. La mayoría de estudios concuerdan que es importante determinar los niveles plasmáticos de la inhibina, ya que puede estar elevada en aproximadamente un $90 \%$ de las yeguas con TCG (MCCUE, 2000, LEBLANC et al., 2003); desafortunadamente en este caso clínico no fue analizada esta hormona, debido a la ausencia de disponibilidad de la prueba en laboratorios especializados del país. 
Aunque para FRAZER (2003) el tratamiento hormonal con GnRH o Gonadotropina Coriónica humana (hCG) suele ser inútil en la mayoría de los casos de agrandamiento ovárico; en este estudio el paciente obtuvo resultados satisfactorios con la aplicación intramuscular de $2.2 \mathrm{mg}$ de $\mathrm{GnRH}$, permitiendo la reducción del tumor en aproximadamente un $30 \%$ de su tamaño. Esta dosis es utilizada principalmente para la ovulación de folículos terciarios en yeguas (BERTONE e HORSPOOL, 2004).

La terapia con $\mathrm{GnRH}$ en neoplasias de ovario ha sido ampliamente documentada en humanos y su administración esta fundamentada en la supresión de $\mathrm{FSH}$ y $\mathrm{LH}$, dos hormonas que promueven el crecimiento y diferenciación celular, cumpliendo un papel importante en la carcinogénesis ovárica (CHOPIN et al., 2002; LIMONTA et al., 2003; MARELLI et al., 2006; FISTER et al., 2008). Igualmente la $\mathrm{GnRH}$ ha sido implicada en la reducción de angiogénesis, crecimiento celular y metástasis de melanoma en humanos (MORETTI et al., 2010).

La remoción quirúrgica del ovario afectado es la única forma de tratamiento para eliminar los cambios comportamentales y permitir el retorno de la ciclicidad del ovario atrofiado (MAURICE, 2006; GÜNDÜZ et al., 2010; MCKINNON e BARKER, 2010). En la mayoría de ocasiones el ovario atrofiado reactiva su función en los próximos 9 meses de realizarse el procedimiento quirúrgico (LEBLANC, et al., 2003). En el caso de la yegua del estudio se observó ciclicidad a los 3 meses de efectuada la cirugía.

Después de la cirugía del paciente la masa tumoral fue enviada para examen histopatológico, que es considerado como el diagnóstico definitivo para TCG (FRAZER, 2003; MAURICE, 2006; MCKINNON e BARKER, 2010). En el examen, el tejido neoplásico estaba constituido por células dispuestas en nidos y sustentadas por un delicado estroma fibrovascular, con características morfológicamente semejantes a las células de la granulosa, concordando con la descripción de FAGUNDES e LIMA (2003), MACLACHLAN e KENNEDY (2004) y LIMA et al., (2011). En la Figura 5 se observa el TCG y sus lesiones microscópicas.

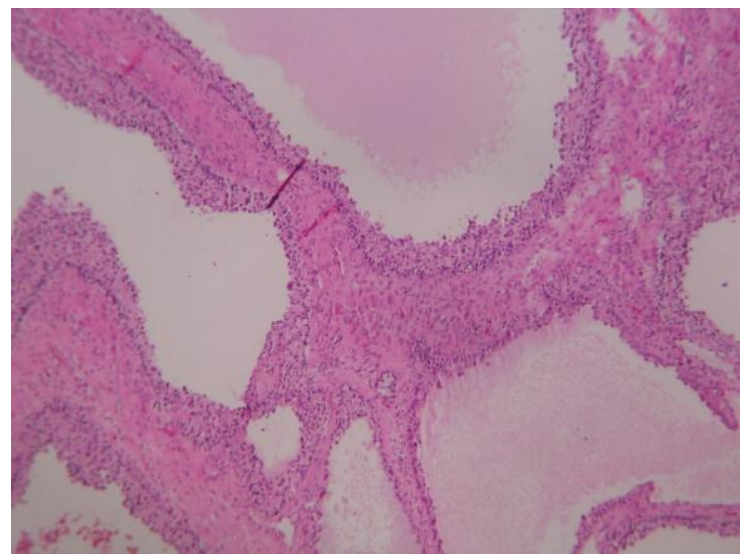


Figura 5. Tumor de células de la granulosa. Múltiples dilataciones quísticas foliculares delimitadas por células neoplásicas y separadas por estroma fibroso. H-E 100X.

\section{Conclusión}

Para el diagnóstico de cualquier problema reproductivo, principalmente los generados por neoplasias, es indispensable realizar un seguimiento al historial clínico del animal e implementar herramientas como la ultrasonografía o pruebas que determinen los niveles hormonales en el plasma sanguíneo, si se sospecha de un tumor que secreta hormonas.

Igualmente es importante que el veterinario encargado del caso siempre recomiende al propietario el envío de muestras de la masa tumoral al laboratorio de histopatología con el fin de conocer el diagnóstico definitivo de la neoplasia.

En el caso de la yeguas con TCG, después de efectuado el tratamiento hormonal y la remoción quirúrgica, el pronóstico es muy favorable y con frecuencia permite que el individuo continué siendo utilizado en meses posteriores para reproducción.

\section{Referencias}

BERTONE, J. J., HORSPOOL, L. J. 2004. Drugs and dosages for use in equines. Págs 367-380 en: Bertone, J. J., Horspool, L. J. Equine clinical pharmacology. Editorial Saunders. London, UK.

CHOPIN, J. B., CHOPIN, L. K., KNOTT, L. M., DE KRETSER, D. M., DOWSETT, K. F. 2002. Unusual ovarian activity in mare preceding the development of an ovarian granulosa cell tumour. Australian Veterinary Journal 80: 32-36.

FAGUNDES, E., LIMA, R. 2003. Patologias do Ovário. Págs 15-39 en: FAGUNDES, E., LIMA, R. Patologia da Reprodução dos Animais Domésticos. Editorial Guanabara Koogan, $2^{a}$ ed. Rio de Janeiro, Brasil.

FISTER, S., SCHLOTAWA, L., GUNTHERT, A. R., EMONS, G., GRUNDKER, C. 2008. Increase of doxubicin-induced apoptosis after knock-down of gonadotropin-releasing hormone receptor expression in human endometrial, ovarian and breast cancer cells. Gynecological Endocrinology 24:24-29.

FOSTER, R. A. 2009. Sistema Reprodutivo da Fêmea. Págs 1263-1315 en: MCGAVIN, M. D., ZACHARY,J. F. Bases da Patologia em Veterinária. Editorial Elsevier, 4⿳亠丷a ed. Rio de Janeiro, Brasil. 
FRAZER, G. S. 2003. Evaluation of Equine Ovary. Págs 260-263 en: FATHMAN, L. Current Therapy in Equine Medicine 5. Editorial Saunders, $5^{\mathrm{a}}$ ed. Misouri, USA.

GÜNDÜZ, M. C., KAŞIKÇI, G., KILIÇARSLAN, R., UÇMAK M., DÜZGÜN, O., TEK, Ç. 2010. Reproductive performance following unilateral ovariectomy for treatment of ovarian tumors in 7 mares. Turkish Journal of Veterinary Animal Sciences. 34: 283-287.

HARTWIG, F. P., SANTOS, R. S., NOGUEIRA, C. E. 2009. Tumor de células da granulosa - Estudo de caso. In: XVIII Congresso de iniciação Científica realizado pela Universidade Federal de Pelotas, Rio Grande do Sul. [Citado en: 25 de septiembre de 2013]; URL Disponible en: $<$ http://www.ufpel.edu.br/cic/2009/cd/pdf/CA/CA 01769.pdf>.

INFANTE, R. M. 1998. Tumores de la granulosa y la teca en 93 yeguas. Revista científica FCV-LUZ 8: 222-228.

KÖNING, H. E., MAIERL, J., LIEBICH, H. G. 2005. Órganos genitales femeninos. Págs. 135-152 en: KÖNING, H. E., LIEBICH, H. G. Anatomía de los Animales Domésticos. Editorial Panamericana, $2^{\mathrm{a}}$ ed. Madrid, España.

LEBLANC, M., LOPATE, C., KNOTTENBELT, D., PASCOE, R. 2003. The Mare. Págs 113-212 en: KONTTENBELT, D. C., LEBLANC, M., LOPATE, C., PASCOE, R. Equine Stud Farm Medicine and Surgery. Editorial Saunders. London, United Kingdom

LIMA, R., FAGUNDES, E., EDWARDS, J. F. 2011. Sistema Reprodutivo Femenino. Págs 797-854 en: Santos, R.L., Alessi, A.C. Patologia Veterinária. Editorial Roca. São Paulo, Brasil.

LIMONTA, P., MORETTI, R. M., MARELLI, M. M., MOTTA, M. 2003. The biology of gonadotropin hormone-releasing hormone: role in the control of tumor growth and progression in humans. Frontiers in Neuroendocrinology 24: 17-27.

MACLACHLAN, N. J., KENNEDY, P. C. 2004. Tumors of the Genital Systems. Páginas 547-606 en: MEUTEN, D. J. Tumors in Domestics Animals. lowa State Press $4^{a}$ ed. lowa, USA.

MARELLI, M., MORETTI, R. M., JANUSZKIEWIEZ-CAULIER, M., MOTTA, M., LIMONTA, P. 2006. Gonadotropin-Realising Hormone $(\mathrm{GnRH})$ Receptors in Tumors: a New Rationale for the Therapeutical Application of GnRH Analogs in Cancer Patients?. Current Cancer Drug Targets 6: 257:269.

MAURICE, K. T. 2006. Diagnosis and surgical removal of a granulosa-theca cell tumor in a mare. The Cananadian Veterinary Journal 46: 644-646.

MCCUE, P.M. 1992. Equine granulosa cell tumors. American Association of Equine Pratitioners 38: 587-593. 
MCCUE, P. M. 2000. Diagnóstico de patologías ováricas. International Veterinary Information Service (IVIS). [Citado en: 21 de septiembre de 2012]; URL Disponible en: <http://www.fveter.unr.edu.ar/Objetos/ivis.pdf>

MCCUE, P. M., ROSER, J. F., MUNRO, C. J., LIU, I. K., LASLEY, B.L. 2006. Granulosa cell tumors of the equine ovary. Veterinary Clinics of North America: Equine Practice 22: 799-817.

MCCUE, P. M. 2009. The problem mare: Diagnosis and Management. VII Curso Internacional de reproducción en equinos. México D.F. [Citado en: 21 de septiembre de 2012]; URL Disponible en: $<$ http://www.remevet.com/pdf/curso int.pdf $>$.

MCKINNON, A. O., BARKER, K. J. 2010. Clinical commentary granulosa theca cell tumors. Equine veterinary education 22: 121-124.

MORA, V., FUENTES, E., GONGORA, A. 2004. Diagnóstico y tratamiento de un tumor de las células de la granulosa en una yegua criolla colombiana. Revista Orinoquía 8: 42-48.

MORETTI, R. M., MAI, S., MARELLI, M., BANI, M. R., GHILARDI, C., GIAVAZZI, R. TAYLOR, D. N. et al. 2010. Dual Targeting of Tumor and Endothelial Cells by Gonadotropin-Releasing Hormone Agonists to Reduce Melanoma Angiogenesis. Endocrionology 151: 4643-4653.

OtALVARO, L. J., CORREA, C. J., VELÁSQUEZ, C. E., MALDONADOESTRADA, J. G. 2009. Falla ovárica en una yegua de paso fino colombiano: tratamiento combinado homeopático antihomotóxico y hormonal. Reporte de caso. Revista Colombiana de Ciencias Pecuarias 22: 665-675.

PINTO, C. R., PACCAMONTI, D. L. 2004. Mare Reproductive pathology. Págs 1039-1049 en: REED, S. M., BAYLY, W. M., SELLON, D. C. Equine Internal Medicine. Editorial Saunders, $2^{a}$ ed. St Louis, USA.

SCHLAFER, D. H., MILLER, R. B. 2007. Female genital system. Páginas 431563 en: MAXIE, M. G. JUBB, KENNEDY, PALMER'S Pathology of domestic animals. Editorial Saunders, $5^{\stackrel{a}{ }}$ ed. Ontario. 